\title{
SELECTIVE EFFECTS IN COSMIC RAYS INDUCED BY COULOMBIAN INTERACTIONS WITH FINITE TEMPERATURE PLASMAS
}

J. Pérez-Peraza

Tata Institute of Fundamental Research, Bombay, India and Instituto de Astronomia, U.N.A.M., Mexico, D.F., Mexico, and S.S. Trivedi

Tata Institute of Fundamental Research, Bombay, India

The role of Coulombian energy losses in cosmic ray physics is generally over simplified by using the Bethe-Block formulation which does not depend explicitly on the tempera-. ture of the medium. The role of low energy particles is usually neglected, as a result of the over estimation of losses when the temperature of the medium is ignored. A deep analysis of Coulombian losses may raise the importance of these particles in the dynamics of the Galaxy. In fact, the deceleration of these particles is determined by charge interchange processes with the target ions and electrons, which energy dependence is roughly the inverse of ionisation losses. Even high energy particles may be subject to this kind of deceleration if the temperature is very high. The consideration of Coulombian losses through all energy ranges with explicit dependence on the temperature has been discussed by Perez and Lara (1979): a fully ionized medium of hydrogen has been assumed to prevail in most of cosmic ray sources. One kind of the implications is the determination of particle composition. It is claimed that a given kind of ion is preferentially accelerated or depleted depending on whether the acceleration is higher or lower than the deceleration rate at the beginning of the acceleration of thermal material. Species which undergo depletion are accelerated only if their energy is higher than that for which both rates are equated $\left(E_{C}, E_{C}^{\prime}\right.$ and $\left.E_{C}^{\prime}\right)$ in such a way that only those of the hot tails of their thermal distributions are effectively accelerated. These will appear depleted relative to other species which are free accelerated because their deceleration rates at low energies are lower than the acceleration rate. It can be noted in the next figures, that if both rates would not intersect at the beginning of the acceleration, they would not join at higher energies because the acceleration rate grows faster with energy than the deceleration rate. Three arbitrary acceleration rates are used for illustration: Fermi-2 nd order ( $\alpha \beta W)$, Betatron or adiabatic heating $\left(\alpha \beta^{2} W\right)$ and shock wave acceleration $(\alpha W)$, where $\alpha, \beta$ and $w$ are the 

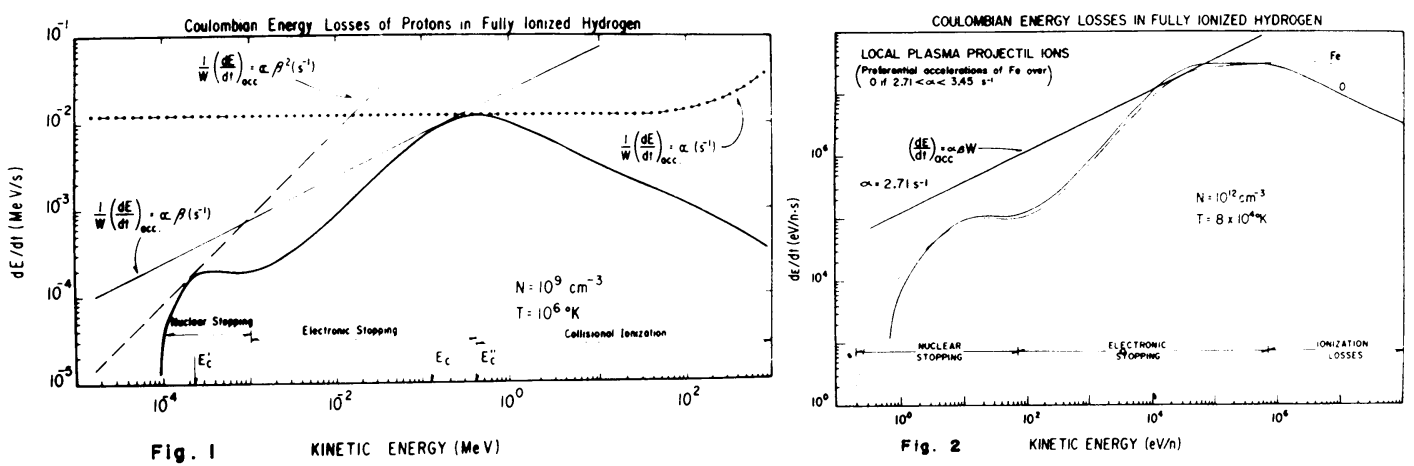

efficiency, the particles velocity and the total energy per nucleon respectively. In Fig.l it can be seen that this selective acceleration relative to Coulombian losses is defined at different energy levels depending on the kind of acceleration involved. Since the main effect of the temperature on the losses at the beginning of the acceleration is through the local charge states of the ions, the sequence of energy losses among different species is highly assorted. This is translated in a great amount of possibilities of particle enhancements and depletions according to the temperature of the source and the kind of acceleration operating therein. If particles under go acceleration in a fully stripped state, the sequence of losses at all energy levels is such that the heavy elements are depleted in relation with the lighter ones; same is the situation, what-ever the initial charge state, for high energy particles in the range of ionisation. It may be concluded, on basis to the observational enhancement of heavy cosmic rays, that hot regions are not likely sources, and that acceleration initiates from thermal energies. On Fig.2 it is illustrated the enhancement of $\mathrm{Fe}$ over $\mathrm{O}$ in solar flare conditions, on basis to the charge states as given by Jordan (1969). If $\alpha<2.71 \mathrm{~s}^{-1}$ both elements would be depleted, whereas if $\alpha>3.45 \mathrm{~s}^{-1}$ both would be preferentially accelerated.

The temperature effect must also be considered in the demodulation of particles fluxes to derive the cosmic ray source abundances within the Galoctogenic or Autogenic hypothesis. The ability of $\mathrm{KeV}$ particles to escape from their sources and propagate in the inhomogeneous interstellar medium must be quantified on the same grounds.

\section{Acknowledgments:}

J.P.P. wish to thank Prof. S. Biswas for his kind hospitality and to the TIFR for financial support.

References:

Jordan, C: 1969, Mon. Not. R. Ast. Soc., 142, 501

Pérez-Peraza, J. and Lara, R: 1979, Proc. of $16^{\text {th }}$ Int. Conf.

Rays Conf., 5, 153 